\title{
Optical Sensor of the Magnetic Field in Electrical Power Engineering
}

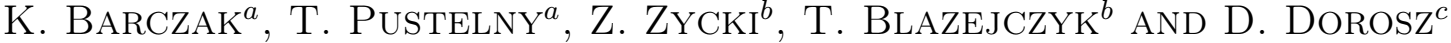 \\ ${ }^{a}$ Department of Optoelectronics, Institute of Physics, Silesian University of Technology \\ Krzywoustego 2, 44-100 Gliwice, Poland \\ ${ }^{b}$ Electrotechnical Institute, Pozaryskiego 28, 04-703 Warsaw, Poland \\ ${ }^{c}$ Department of Optical Radiation, Białystok Technical University, Wiejska 45, 15-950 Białystok, Poland \\ The authors of the presented paper deal with investigations concerning applications of the optical fiber sensor \\ of the magnetic field in electric DC engines with permanent magnets. The aim of the sensor is to measure the \\ state of magnetization of permanent magnets (basing on the measurements of magnetic field induction). The \\ knowledge of the state of magnetization helps to decide whether permanent magnets need to be additionally \\ magnetized in order to prevent damages of the whole engine. This can be done automatically, even when the \\ engine is running. For the purpose the Faraday magnetooptic effect is used. The presented investigations concern \\ an optical fiber sensor of the magnetic field provided with a glass rod characterized a high value of the Verdet \\ constant as the sensing element. Thanks to the application of optical fibers the connection of the sensor head with \\ the whole measuring system is constructed of insulating material without using of electrical connections. \\ PACS numbers: 42.70.-a, 42.81.-i, 42.79.Pw, 78.20.Ls
}

\section{Introduction}

Direct current engines with permanent magnets are apt to be demagnetized, for instance due to short-circuit or overcharging. Usually this is only a partial demagnetization, in results of which the electrical engine loses its operational properties (and thus its efficiency). Partially demagnetized engines ought to be demagnetized as soon as possible in order to prevent a break-down of the engine. For this purpose coils may be installed in the engine at the poles of the permanent magnets [1]. At the instant of demagnetization the coils can demagnetize the magnets by an electric current impulse of some milliseconds. Such a solution permits to improve the state of magnetization of the permanent magnets while the engine is running. One of the basic elements of such a system is the sensor operating and controlling the state of magnetization of the permanent magnets [2-4]. Thanks to the cooperation of the Institute of Electrical Engineering with the Department of Optoelectronics at the Silesian University of Technology investigations could be started concerning an optical fibre sensor of the magnetic field operating basing on Faraday's magnetooptical effect [5]. An advantage of this sensor is that light can be utilized as a carrier of information about the induction of the magnetic field. The beam of light that is introduced and carried out in an optical fibre, is insensible to electromagnetic disturbances [6-8].

\section{Optical sensor of the magnetic field}

This sensor used optical fibers for the purpose of transmitting an optical signal. For the construction of the sensing element a glass rod was applied with a diameter of $1.8 \mathrm{~mm}$ and a length of $34 \mathrm{~mm}$, consisting of multicomponential glass with a refractive index of 1.65 . The measured Verdet constant of this glass amounted to
$4.34 \mathrm{rad} /(\mathrm{T} \mathrm{m})$ at an optical wavelength of $635 \mathrm{~nm}$. At both ends of the rod there were stuck polarizers and next were fastened the input and output PCS optical fibers (Fig. 1).

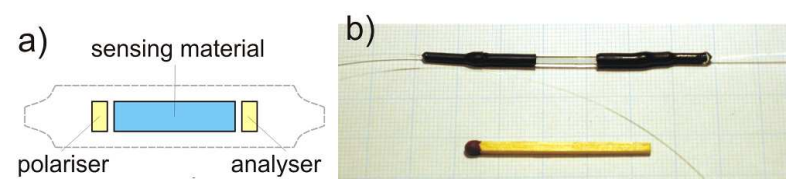

Fig. 1. Structure of the optical sensor of the magnetic field: (a) its scheme, (b) its photo.

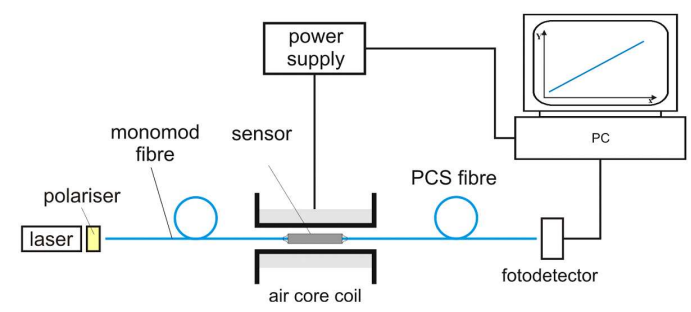

Fig. 2. Test stand.

The sensor was tested in the configuration presented in Fig. 2.

At the output a silicon photodetector was used because changes in the state of polarization were analyzed inside the sensing element (the polarizer at the output plays the role of an analyzer). In this way the entire system was considerably simplified, particularly — its detective part (Figs. 3 and 4).

An important of the performed investigations is the determination of the optimal localization of the sensor in the electric engine. The magnetooptical effect in the 


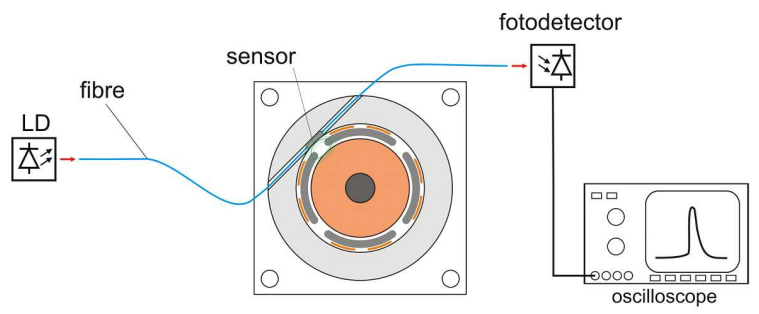

Fig. 3. Measuring stand with an electric engine.

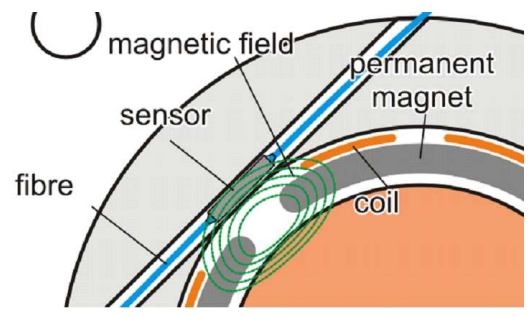

Fig. 4. Example of how to localize the sensor in the electrical engine.

material of the sensor (Fig. 1a) achieves its maximum value when the external magnetic field is parallel with the direction of the ray of light passing through the sensing element. It is the fundamental criterion in the proper choice of the optical localization of the sensor in the electrical engine. The basic problem of this optimization are connected results from the distribution of the magnetic field inside the tested engine. Practically, the entire flux of magnetic field induction $B$ is contained in the magnetic material (stator, rotor, permanent magnets) due to its low reluctance. In the air-gaps (where the reluctance is high), this flux is usually perpendicular or nearly perpendicular to the edges of the air-gap, if this is the only "way" to flow the flux. Leaving the effects of saturation of the magnetic material out of account (not stipulated in the region of localization of the sensor), when the path of the flux can be "shut" by the magnetooptic material, then the magnetic field in the air-gap is imperceptible. This problem must be faced by experts from the Institute of Electrical Engineering, who are eager to find its optimal solution. The proposed solution of localizing the sensor (Figs. 3 and 4) is merely a demonstrative one and cannot be theoretically analyzed (analysis of the field distribution of the field), nor experimentally verified.

\section{Experimental results}

Previous to the construction of the sensor head (Fig. 4) the magnetooptical effect in the sensor glass was investigated. The achieved results have been presented in Fig. 5. As has already been mentioned, the Verdet constant of this material amounted to $4.34 \mathrm{rad} /(\mathrm{T} \mathrm{m})$ at a wavelength of $635 \mathrm{~nm}$. Due to the short path of propagation of the light $(34 \mathrm{~mm})$ the rotatory polarization is slight (1.10). The recorded signal was subjected to some fluc- tuations, which in Fig. 5 is visible in the form of inclination of the whole characteristics. These changes did not prevent the correct determination of the Verdet constant. The fluctuations were caused by changes of the state of polarization in the single-mode waveguide introducing the optical energy into the sensor.

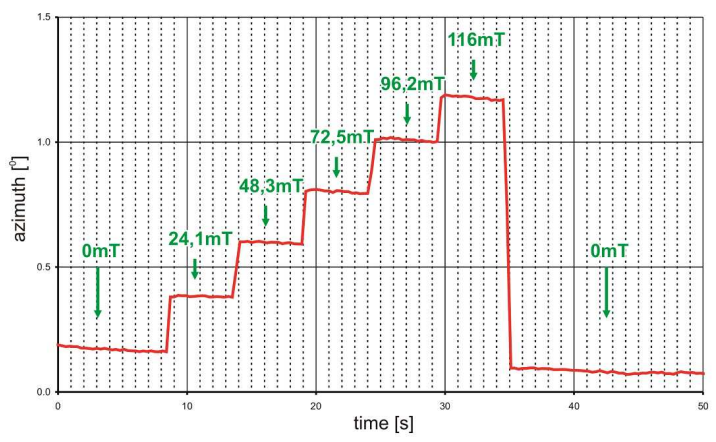

Fig. 5. Magnetooptical effect in the glass root, of which the sensor is made.

The response of the sensor in the stand presented in Fig. 2 is to be seen in Fig. 6. The signal is distinct thanks to the adequate positioning of the polarizer and analyzer in the head of the sensor (Fig. 1a). The diagram presents the response of the sensor to two rectangular impulses of the magnetic field, lasting ten second each, with the values $116 \mathrm{mT}$, as a function of time.

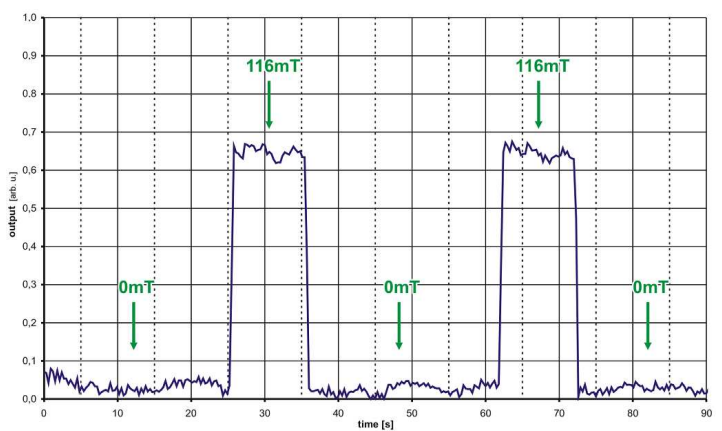

Fig. 6. Response of the sensor to the magnetic field.

\section{Conclusions}

The performed investigations indicate distinctly that the presented sensor based on volumetric glass may be applied in measurements of the magnetic field in an electrical engines. Its main advantages are:

- small geometrical dimensions,

— insensibility to vibrations and bending of the optical fibers,

- simple and cheap method of detecting optical signals,

- compact structure. 
This sensor is planned to be further developed making use of other, more sensitive magnetooptical materials with a Verdet constant amounting to $30 \mathrm{rad} /(\mathrm{T} \mathrm{m})$, which will permit to shorten the sensor element and to ensure conditions approximating pointwise measurements of the values of the magnetic field induction.

\section{Acknowledgments}

This work is financed as the grant of Ministry of Science and Higher Education No. N510 387735.

\section{References}

[1] K. Iwamoto, Appl. Opt. 29, 375 (1990).
[2] T. Błażejczyk, Z. Zycki, Przeglad Elektrotechniczny 1, 91 (2007) (in Polish).

[3] K. Barczak, T. Pustelny, D. Dorosz, J. Dorosz, Europ. Phys. J. - Special Topics 154, 11 (2008).

[4] K. Barczak, T. Pustelny, Z. Zycki, T. Blazejczyk, Acta Phys. Pol. A 116, 250 (2009).

[5] F. Ratajczyk, Birefringence and Optical Polarisation, Wrocław University of Technology Publisher, Wrocław 2000.

[6] D. Dorosz, K. Barczak, T. Pustelny, J. Dorosz, Acta Phys. Pol. A 114, A-61 (2008).

[7] K. Barczak, T. Pustelny, D. Dorosz, J. Dorosz, Acta Phys. Pol. A 116, 247 (2009).

[8] D. Dorosz, M. Kochanowicz, J. Dorosz, Acta Phys. Pol. A 116, 298 (2009). 